\title{
CONDUCTAS FRENTE AL VIRAJE DE LA PRUEBA DE TUBERCULINA EN ESTUDIANTES DE MEDICINA DE UNA UNIVERSIDAD DE LIMA, PERÚ
}

\author{
Lesly Calixto-Aguilar ${ }^{1, a}$, Martiel Manrique-Zegarra ${ }^{1, a}$, Eduardo Gotuzzo-Herencia ${ }^{2,3, b}$, \\ Frine Samalvides-Cuba ${ }^{2,3, c}$
}

\begin{abstract}
RESUMEN
El objetivo del estudio fue determinar las conductas frente al viraje de la prueba de tuberculina (PPD) y las razones del inicio o no del tratamiento de la infección latente tuberculosa (TILT) en estudiantes de Medicina en una Universidad de Lima, Perú; 548 participantes Ilenaron un cuestionario; se obtuvo que 6,7\% fueron positivos al ingreso a la universidad y $11,1 \%$ fueron conversores recientes; de este grupo $55,7 \%$ no iniciaron TILT y la principal razón fue "no hubo explicación". De los conversores recientes, la mayoría de alumnos que sí iniciaron el TILT, eran mayores de 22 años $(p=0,01)$ y respondieron correctamente las preguntas sobre conocimientos generales $(p=0,04)$. Es resaltante que la tasa de conversión de PPD fue más alta a la reportada en la literatura y que la mayoría de estudiantes no siguió la profilaxis por falta de información.
\end{abstract}

Palabras clave: Tuberculosis Latente, profilaxis, conducta, estudiantes de medicina (fuente: DeCS BIREME).

\section{BEHAVIORS IN RESPONSE TO THE TUBERCULIN SKIN TEST CONVERSION IN MEDICAL STUDENTS FROM A UNIVERSITY IN LIMA, PERU}

\begin{abstract}
The aim of this study was to determine the behaviors in response to tuberculin skin test (PPD) conversion and the reasons for starting or not starting treatment for latent tuberculosis infection (TILT) among medical students at a university in Lima, Peru. A total of 548 participants completed a questionnaire; of them, $6.7 \%$ tested positive on university admission and $11.1 \%$ were recent converters. A total of $55.7 \%$ did not start TILT and had no explanation. Of the recent converters, most students who did start TILT were $>22$ years old $(p=0.01)$ and correctly answered general knowledge questions $(p=0.04)$. It is important to note that the PPD conversion rate was higher than that reported in the literature and that most students did not follow the treatment prophylaxis because of a lack of information.
\end{abstract}

Key words: Latent Tuberculosis, prophylaxis, behavior, Medical Students (source: MeSH NLM).

\section{INTRODUCCIÓN}

La tuberculosis (TB) constituye un importante problema de salud global, siendo la segunda causa de muerte por enfermedades infecciosas (1). Según el último informe de la Organización Mundial de la Salud, en el 2013, a nivel mundial se registró una prevalencia de 159 casos por 100000 habitantes, una incidencia de 126 casos por 100000 habitantes y una tasa de mortalidad de 16 casos por 100000 habitantes ${ }^{(1)}$. En el Perú, en el 2012 se reportó una incidencia de tuberculosis de 95 casos por 100000 habitantes y una mortalidad de 5,1 por 100000 habitantes, donde la capital, Lima, alberga el $58 \%$ de los $\operatorname{casos}^{(2)}$.

Los ejes más importantes para la prevención y control de la enfermedad son la detección precoz de casos, su diagnóstico, su tratamiento adecuado, la realización de la prueba de tuberculina (PPD) y el tratamiento de la infección latente ${ }^{(1)}$. En cuanto a este último aspecto, estudios longitudinales sugieren que aproximadamente entre el 5 y $10 \%$ de las personas con infección latente desarrollarán la enfermedad entre uno y dos años tras adquirir la infección, mientras que otro $5 \%$ lo hará a lo

\footnotetext{
Facultad de Medicina, Universidad Peruana Cayetano Heredia. Lima, Perú.

Instituto de Medicina Tropical Alexander von Humboldt, Universidad Peruana Cayetano Heredia. Lima, Perú,

Hospital Nacional Cayetano Heredia. Lima, Perú.

Médico cirujano; ${ }^{\mathrm{b}}$ médico especialista en Enfermedades Infecciosas y Tropicales; ${ }^{\mathrm{c}}$ médico infectólogo, magíster en Epidemiología Clínica.

* Los datos de este estudio forman parte de la tesis para obtener el grado de bachiller en Medicina Humana

Recibido: 27/06/2015 Aprobado: 09/03/2016
} 
largo de la vida ${ }^{(3)}$. Es por ello que en este grupo de convertores recientes está indicado el tratamiento de la infección latente. Sin embargo, diversos estudios muestran que la adherencia al tratamiento es subóptima, incluso en el personal de salud ${ }^{(4,5)}$. Mientras que en la población general se han encontrado como factores relacionados a esto, la falta de medicamentos, efectos secundarios, el uso de alcohol y drogas, una inadecuada educación sobre el problema de salud; en tanto que en estudiantes de Medicina se encontraron asociaciones con el sexo y el nivel socioeconómico ${ }^{(6)}$.

El objetivo de este estudio fue determinar las conductas frente al viraje de la prueba de tuberculina (PPD) y las razones del inicio o no del TILT en estudiantes de Medicina en una Universidad de Lima, Perú.

\section{EL ESTUDIO}

Estudio transversal, realizado durante enero a junio del año 2013, con alumnos de la facultad de Medicina de la Universidad Peruana Cayetano Heredia, Perú, desde el segundo hasta el séptimo año de estudios. El protocolo de la investigación fue aprobado por el Comité de Ética en Investigación de la Universidad Peruana Cayetano Heredia. Todos los participantes fueron debidamente informados, y participaron voluntariamente, previa firma del consentimiento informado.

Se usaron dos cuestionarios: el cuestionario 1 fue elaborado por un grupo de expertos, médicos infectólogos, epidemiólogos e investigadores en el campo, contó con una fase piloto que incluyó a 50 estudiantes de una facultad de Medicina Humana, y su versión final incluye datos demográficos, preguntas de conocimientos generales sobre tuberculosis y preguntas relacionadas a la conversión del PPD de TB. El cuestionario 2 tenía como objetivo describir las razones por las cuales algunos estudiantes no desearon participar.

Los datos fueron ingresados en una tabla en Microsoft Excel 2007 (Microsoft, US) y fueron analizados usando el programa STATA v12.0 (StataCorp, US). Se hizo un análisis descriptivo de las variables demográficas, conocimientos generales y conductas frente a la conversión del PPD. Además, se exploraron las relaciones entre el grupo de estudiantes que inició y no inició el TILT y las variables edad, sexo, año de estudios y nivel de conocimientos, mediante las pruebas chi cuadrado y t de Student.

Se definió operacionalmente como alumno a un estudiante de la Facultad de Medicina de una Universidad de Lima, Perú, que curse entre el segundo y séptimo año de estudios, en el período de recolección de datos. Los años de estudios preclínicos se definen como segundo y tercer año. El convertor reciente es aquel individuo que ha experimentado un viraje de negativo (diámetro de pápula $<10 \mathrm{~mm}$ ) a positivo (diámetro de pápula $\geq 10 \mathrm{~mm}$ ) en la reacción tuberculínica (PPD), incrementándose el diámetro como mínimo entre 6 y $10 \mathrm{~mm}$, cuya medida previa es de un período máximo de 2 años. Siendo la lectura realizada 48 a 72 h después ${ }^{(7,8)}$, y cuya prueba de PPD haya sido realizada en un programa de atención médica integral de salud, el cual no pertenece a la Estrategia Nacional de Prevención y Control de la Tuberculosis (ESN-PCT).

Finalmente, la conducta de los estudiantes se definió a través del cuestionario, con las preguntas sobre la conversión del PPD y el TILT.

\section{HALLAZGOS}

El estudio comprendió al total de alumnos (734) entre el segundo y último año de estudios de una facultad de Medicina de una Universidad de Lima, Perú. De ellos, $548(75 \%)$ accedieron a participar, siendo la media de edad 21,10 años (DE \pm 2 ); $53,7 \%$ fueron varones, $47,8 \%$ se encontraban cursando años de estudios preclínicos y $25,9 \%$ respondieron correctamente las preguntas de conocimiento general; de este último grupo, la mayoría $(97,2 \%)$ pertenecía a años de estudios clínicos.

Del total de participantes, $37(6,7 \%)$ fueron positivos desde el primer año de estudios, $61(11,1 \%)$ fueron convertores recientes; siendo $18 \%$ la prevalencia de la infección latente. En este último grupo, la media de la edad fue 22,8 años (DE \pm 2 ), 50,8\% fueron varones, $68,9 \%$ convirtieron en años de estudios preclínicos, y un $55,7 \%$ no siguieron el TILT.

En el grupo de convertores recientes que sí iniciaron el TILT, la media de la edad fue de 23,3 (DE $\pm 1,4$ ); $13(48,1 \%)$ estudiantes fueron varones; $19(70,4 \%)$ convirtieron en años de estudios preclínicos; 18 (66,7\%) respondieron de manera correctas las preguntas de conocimientos y la principal razón por la que iniciaron el TILT fue "me lo indicó el programa" $(44,4 \%)$, seguido por "leí que era lo que se debía hacer" $(22,2 \%)$ y "me lo recomendó algún profesor" $(19,4 \%)$ (Tabla 1).

En el grupo de convertores recientes que no inició el TILT, la media de edad fue de 22,4 (DS $\pm 1,5) ; 18(52,9 \%)$ participantes fueron varones; $23(67,6 \%)$ convirtieron en los años de estudios preclínicos; 20 (58,8\%) respondieron de manera incorrecta las preguntas de conocimientos y la principal razón por la que no iniciaron el TILT fue "no hubo explicación" (46,5 \%). La segunda razón fue la opción "por temor a los efectos adversos" $(20,5 \%)$, seguido por la razón "porque estaba tomando otra medicina" $(13,9 \%)$ (Tabla 1$)$. 
Tabla 1. Razones por las cuales los convertores recientes iniciaron o no el tratamiento de la infección latente tuberculosa

\begin{tabular}{|c|c|c|}
\hline & $\mathbf{n}$ & $\%$ \\
\hline \multicolumn{3}{|l|}{$\begin{array}{l}\text { Razón por la que siguió el tratamiento de la } \\
\text { infección latente tuberculosa }(n=27)\end{array}$} \\
\hline Me lo indicó el programa & 16 & 44,4 \\
\hline Leí que era lo que se debía hacer & 8 & 22,2 \\
\hline $\begin{array}{l}\text { Me lo recomendó algún profesor de la } \\
\text { universidad }\end{array}$ & 7 & 19,4 \\
\hline Me lo indicó el neumólogo & 5 & 13,8 \\
\hline \multicolumn{3}{|l|}{$\begin{array}{l}\text { Razón por la que no siguió tratamiento de } \\
\text { la infección latente tuberculosa }(n=34)\end{array}$} \\
\hline No hubo explicación & 20 & 46,5 \\
\hline Temor a los efectos adversos & 7 & 20,5 \\
\hline $\begin{array}{l}\text { Porque estaba tomando otra medicina o } \\
\text { recién había acabado con esta }\end{array}$ & 6 & 13,9 \\
\hline Porque si la seguía no podía tomar alcohol & 4 & 9,3 \\
\hline Porque tuve hepatitis viral hace tiempo & 1 & 2,3 \\
\hline Seguimientos de tratamientos alternos & 0 & 0 \\
\hline Podría inducirme resistencia & 0 & 0 \\
\hline Otras & 5 & 11,6 \\
\hline
\end{tabular}

Además, se exploraron las relaciones entre las diferentes variables (Tabla 2). En el grupo de los convertores recientes, los estudiantes que sí siguieron el TILT tenían una edad mayor a 22 años $(p=0,01)$ y respondieron correctamente las preguntas sobre conocimientos generales $(p=0,04)$, con diferencias significativas. Mientras que no se encontró una relación significativa entre los estudiantes que convirtieron en años de estudios preclínicos y el seguimiento del TILT $(p=0,8)$.

\section{DISCUSIÓN}

El presente estudio encontró que existe poco conocimiento sobre la tuberculosis en estudiantes de Medicina, ya que solo $25,9 \%$ de ellos respondieron correctamente, porcentaje mucho menor a lo encontrado en otros estudios, como uno realizado en Europa, que mostró un porcentaje promedio de respuestas correctas de $56,6 \%$, encontrando, además, que los estudiantes de último año y aquellos que habían recibido la prueba de tuberculina, tuvieron un mayor conocimiento sobre el diagnóstico y tratamiento de esta enfermedad ${ }^{(9)}$.

En cuanto a la infección latente tuberculosa, una reciente revisión sistemática registró un riesgo anual de $2,9 \%$ en países de baja incidencia de tuberculosis y de $7,2 \%$ en países de alta incidencia ${ }^{(10)}$. Sin embargo, en la población de trabajadores del área de salud, los porcentajes son variables; en Italia se encontró una prevalencia de infección latente de $1,4 \%{ }^{(11)}$; mientras que en India, se encontró una tasa de incidencia anual de infección en enfermeras de $7,8 \%$, lo cual fue significativamente mayor a su promedio nacional $(1,5 \%)^{(12)}$. Por otro lado, se han encontrado mayores prevalencias en países de alta incidencia, llegando incluso a $47,8 \%$ entre estudiantes del área de salud ${ }^{(13)}$, en el presente estudio se encontró una prevalencia de $18 \%$.

Luego, en cuanto a la conversión de la prueba de tuberculina, se evidenció un porcentaje de conversión de $11,1 \%$, mientras que en otros estudios en Sudamérica, que utilizaron criterios de conversión similares al nuestro, los porcentajes variaron entre $4,6 \%$ en Brasil, y $16,2 \%$ en Colombia ${ }^{(7)}$. En Perú, un trabajo tipo cohorte registró una incidencia de conversión de 12,4 por cada 100 personas-año de observación ${ }^{(8)} y$, específicamente, en la población de trabajadores del área de salud, en un Hospital de Lima, Perú, en el 2006 se encontró una tasa de conversión de $1,1 \%{ }^{(14)}$. Finalmente, un estudio descriptivo realizado en una universidad de Lima, entre el 2002 y 2006, determinó que el porcentaje de conversión anual era de 5,8\%; y que la mayoría de los alumnos que viraron pertenecían a los primeros años de estudios, eran mujeres, mayores de 18 años y no iniciaron el TILT $(60,4 \%)^{(6)}$; resultados similares a los de este trabajo.

Con relación al año de conversión, un trabajo realizado en Europa presentó resultados controversiales; en algunos

Tabla 2. Diferencias en el seguimiento o no del tratamiento para la infección latente tuberculosa de los convertores recientes y edad, año de conversión y respuestas correctas o no sobre conocimientos

\begin{tabular}{|c|c|c|c|c|c|}
\hline & \multicolumn{2}{|c|}{$\begin{array}{l}\text { Siguió tratamiento para infección } \\
\text { latente tuberculosa }(n=27)\end{array}$} & \multicolumn{2}{|c|}{$\begin{array}{l}\text { No siguió tratamiento para } \\
\text { infección latente tuberculosa } \\
\qquad(n=34)\end{array}$} & \multirow[b]{2}{*}{ Valor $p$} \\
\hline & $\mathbf{n}$ & $\%$ & $\mathbf{n}$ & $\%$ & \\
\hline \multicolumn{6}{|c|}{ Años de conversión } \\
\hline Preclínicos & 19 & 45,2 & 23 & 54,8 & 0,80 \\
\hline Clínicos & 8 & 42,1 & 11 & 57,9 & \\
\hline \multicolumn{6}{|l|}{ Edad } \\
\hline$\leq 22$ años & 6 & 25,0 & 75 & 18,0 & 0,01 \\
\hline$>22$ años & 21 & 56,8 & 16 & 43,2 & \\
\hline \multicolumn{6}{|c|}{ Respuestas a preguntas } \\
\hline Correctamente & 18 & 56,2 & 14 & 43,8 & 0,04 \\
\hline Incorrectamente & 9 & 31,0 & 20 & 68,9 & \\
\hline
\end{tabular}


realizados en estudiantes de Enfermería, Medicina y Terapia Física se mencionan que existen mayores tasas de conversión en los años de estudio clínicos; mientras que en otros se describe que no existen diferencias ${ }^{(15)}$. En el Perú, un estudio encontró que la exposición a pacientes con tuberculosis es un evento común en estudiantes en el ambiente hospitalario, donde el riesgo de adquirirla en el hospital es de 2 a 50 veces más alta que en la comunidad ${ }^{(16)}$. Sin embargo, en el presente estudio, la mayoría de estudiantes convirtieron durante años de estudios preclínicos. Se puede plantear que estos hallazgos pueden estar relacionados a diversos factores, como son, la alta incidencia de la enfermedad, sobre todo en Lima; la exposición en ambientes poco ventilados, como es el transporte público, lo cual se observó en un estudio realizado en el 2015 en Lima, que encontró una asociación entre el uso de buses/minibuses con el riesgo de tuberculosis (OR: 3,5 IC 95\%:1,6-7,6) (17). Así como también, la realización de trabajos comunitarios en años de estudios preclínicos, donde existe exposición de los alumnos a poblaciones numerosas en zonas de mayor incidencia de tuberculosis.

En relación al TILT, un estudio multicéntrico en Estados Unidos y Canadá encontró que solo el 53\% de los participantes completó el TILT (18); y cifras aun más alarmantes se encontraron en un estudio realizado por Soto, et al., que evidenció que $43,5 \%$ de los convertores iniciaron el TILT y de estos, $63 \%$ no lo completó ${ }^{(19)}$. De este modo, se han realizado diversos estudios con el fin de encontrar los factores relacionados a la falta de adherencia al TILT, en su mayoría analizando los factores demográficos asociados. En el presente estudio, se encontró que los convertores que siguieron el TILT eran principalmente mayores de 22 años y habían respondido correctamente las preguntas sobre conocimientos generales, yrelaciones significativas. Mientras que Allinger, et al., en una población de latinos inmigrantes en Estados Unidos, no encontraron una relación entre la edad, años de educación, sexo, estado civil, ocupación y la falta de adherencia al tratamiento ${ }^{(20)}$. Sin embargo, encontraron una relación significativa con la aparición de efectos adversos ${ }^{(20)}$. Fiske, et al. encontraron que las principales razones para no completar el TILT fueron la pérdida de seguimiento (46\%) y la falta de adherencia $(25 \%)^{(18)}$. No obstante, ningún estudio se centró en el grupo de estudiantes del área de la salud en un país de alta incidencia. Es así que en el presente estudio se obtuvo que las principales razones por las que los convertores no iniciaron el TILT fueron, en primer lugar, la falta de explicación por parte del programa, seguido del temor a efectos adversos y la toma de otra medicina en el momento de conversión. Estos hallazgos remarcan la importancia de implementar cambios en los programas para la prevención de tuberculosis en estudiantes de Medicina, ya que la falta de conocimientos acerca de la infección, así como una adecuada explicación sobre las ventajas y desventajas del cumplimiento del TILT, podrían ser la causa de una baja adherencia a esta y de un posible desarrollo de la enfermedad en un futuro.

Contribuciones de autoría: LCA, MMZ y EG han participado en la concepción del artículo. Mientras que la recolección de datos y la redacción estuvo a cargo de LCA y MMZ. Además, FS realizó el análisis de datos, y conjuntamente con EG realizaron la asesoría técnica y administrativa y la aprobación de la versión final.

\section{Fuente de financiamiento: autofinanciado}

Conflictos de interés: no existe conflictos de interés en la publicación del estudio.

\section{REFERENCIAS BIBLIOGRÁFICAS}

1. World Health Organization. Global Tuberculosis Report 2014. Switzerland: WHO Press; 2014. p. 20-35.

2. World Health Organization. Global Tuberculosis Report 2013. Switzerland: WHO Press; 2013. 185 p.

3. American Thoracic Society. Targeted Tuberculin Testing and Treatment of Latent Tuberculosis Infection. Am J Respir Crit Care Med. 2000;161(4 Pt 2):S221-47.

4. Acosta J, Parra E, Rivera R. Viraje de la prueba de tuberculina (PPD) en estudiantes de medicina de la Universidad El Bosque luego de iniciar sus prácticas clínicas. Rev. ECM. 2003;8(1):49-58.

5. Fernández D. Riesgo de adquirir infección tuberculosa en estudiantes de medicina [Tesis bachiller]. Lima: Facultad de Medicina, Universidad Peruana Cayetano Heredia; 1987.

6. Samalvides F, Castañeda E, Poggi L. Cumplimiento al Tratamiento profiláctico de tuberculosis en estudiantes de la Facultad de Medicina Alberto Hurtado. [Tesis bachiller]. Lima: Facultad de Medicina, Universidad Peruana Cayetano Heredia; 2008.

7. Pérez-Lu JE, Cárcamo CP, García PJ, Bussalleu A, Bernabé-Ortiz A. Tuberculin skin test conversion among health sciences students: A retrospective cohort study. Tuberculosis (Edinb). 2013;93(2):257-62. doi: 10.1016/j. tube.2012.10.001.
8. Hohmuth BA, Yamanija JC, Dayal AS, Nardell E, Salazar JJ, Smith Frawzi MC. Latent tuberculosis infection: risks to health care students at a hospital in Lima, Peru. Int J Tuberc Lung Dis. 2006;10(10):1146-51.

9. Laurenti P, Federico B, Raponi M, Furia G, Ricciardi W, Damiani G. Knowledge, experiences, and attitudes of medical students in Rome about tuberculosis. Med Sci Monit. 2013;19:865-74. 10.12659/ MSM.889515.

10. Joshi R, Reingold AL, Menzies D, Pai M. Tuberculosis among health-care workers in low- and middle-income countries: a systematic review. PLoS Med. 2006;3:e494. doi: http://dx.doi. org/10.1371/journal.pmed.0030494 
11. Durando P, Sotgiu G, Spigno F, Piccinini M, Mazzarello G, Viscoli $\mathrm{C}$, et al. Latent tuberculosis infection and associated risk factors among undergraduate healthcare students in Italy: a cross-sectional study. BMC Infect Dis. 2013;13:443. doi: 10.1186/1471-2334-13-443.

12. Christopher DJ, James P, Daley $\mathrm{P}$, Armstrong L, Isaac BT, Thangakunam $\mathrm{B}$, et al. High Annual Risk of Tuberculosis Infection among Nursing Students in South India: A Cohort Study. PLoS One. 2011;6(10):e26199. doi: 10.1371/journal.pone.0026199.

13. Christopher DJ, Daley P, Armstrong L, James P, Gupta R, Premkumar P, et al. Tuberculosis infection among young nursing trainees in south India. PLoS ONE. 2010; 5(4):e10408. doi: 10.1371/journal.pone.0010408.

14. Schablon A, Beckmann G, Harling M, Diel R, Nienhaus A. Prevalence of latent tuberculosis infection among health care workers in a hospital for pulmonary diseases. J Occup Med Toxicol. 2009;4:1. doi: 10.1186/1745-6673-4-1.

15. Montagna MT, Napoli C, Tafuri S, Agodi A, Auxilia F, Casini B, et al. Knowledge about tuberculosis among undergraduate health care students in 15 Italian universities: a crosssectional study. BMC Public Health. 2014;14:970. doi: 10.1186/14712458-14-970.

16. Accinelli R, Noda J, Bravo E, Galloso M, López L, Da Silva J, et al. Enfermedad tuberculosa entre trabajadores de salud. Acta Med Per. 2008;26(1):35-47.

17. Zamudio C, Krapp F, Choi HW, Shah L, Ciampi A, Gotuzzo E, et al. Public transportation and tuberculosis transmission in a high incidence setting. PLoS One. 2013;10(2): e0115230. doi: 10.1371/journal.pone.0115230.

18. Fiske CT, Yan FX, Hirsch-Moverman Y, Sterling TR, Reichler MR, Tuberculosis Epidemiologic Studies Consortium Task Order 2 Team. Risk factors for treatment default in close contacts with latent tuberculosis infection. Int $\mathrm{J}$ Tuberc Lung Dis. 2014;18(4):421-7. doi: 10.5588/ ijtld.13.0688.

19. Soto A, Huablocho P, Ulloque J. Early conversion of tuberculin skin test in medical students who begin hospital practices. Braz J Infect Dis. 2013;17(3):383-4. doi: 10.1016/j. bjid.2012.10.027.

20. Allinger RL, Black P, Nguyen N, Lasus H. Predictors of adherence to latent tuberculosis infection therapy in Latino immigrants. J Community Health Nurs. 2007;24(3):191-8. doi: $10.1080 / 07370010701429637$.

Correspondencia: Lesly Sttefany Calixto Aguilar Dirección: Avenida José de la Riva Agüero 728. Dpto 501 - San Miguel. Lima, Perú Teléfono: (+511) 942663631

Correoelectrónico:lesly.calixto.a@upch.pe

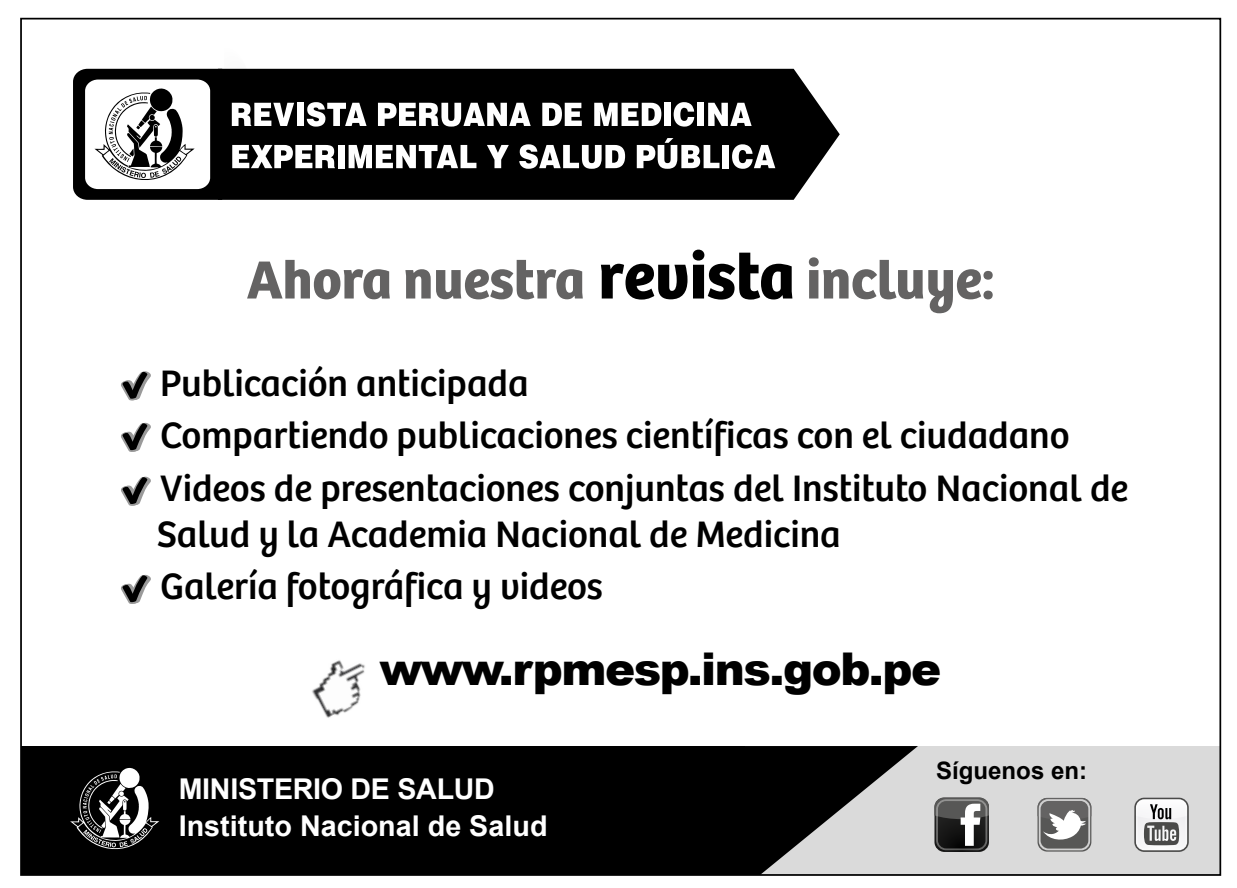

